\title{
Über die Synthese der Hämatinsäure und ūber die Oxydation des Hämatins.
}

\author{
Von \\ William Küster und Johannes Weller.
}

(Mitteilung aus dem Laboratorium für organische Chemie der Technischen Hochschule Stuttgart.)

(Der Redaktion zugegangen am 2. März 1917.)

Die Synthese der Hämatinsäure ist von uns bisher nur in einer kurzen Abhandlung beschrieben worden. ${ }^{1}$ ) Wir kommen auf dieselbe nunmehr eingehender zurück, zumal wir die bereits mitgeteilten Beobachtungen ergänzen können.

Als Ausgangspunkt für die Synthese der Hämatinsäure diente der Acetylglutarsäureester; durch Anlagerung von Blausäure und Verseifung der erhaltenen Verbindung wurde ein amorpher Stoff erhalten, der als ein Gemisch racemischer Oxysäuren mit ihren Lactonen durch die Analyse von Salzen und einem Methylester charakterisiert werden konnte. Durch Abspaltung von Wasser, die durch Erhitzen unter vermindertem Druck erreicht wurde, ging daraus fast quantitativ Hämatinsäure hervor, so daß folgender Verlauf der Reaktionen festgelegt werden konnte:<smiles>COC(=O)C(CCC(=O)O)OC</smiles><smiles>CCCCCCC(=O)OCCCCCC(=O)OCC</smiles><smiles>CC(O)C(CCC(=O)O)C(=O)O</smiles>

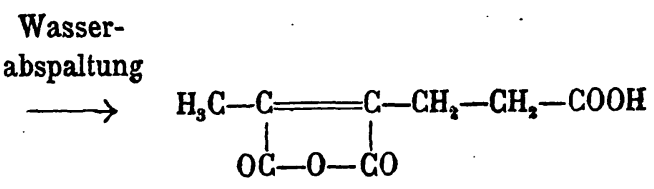

1) Ber. d. d. chem. Ges., Bd. 47, S. 532 (1914).

Huppe-Seyler's Zeitschrift r. physiol. Chemie. IC. 
Als Nebenprodukt trat Acetylbuttersäure auf, entstanden aus Teilen des Acetylglutarsäureesters, die sich der Kondensation mit Blausäure entzogen hatten,

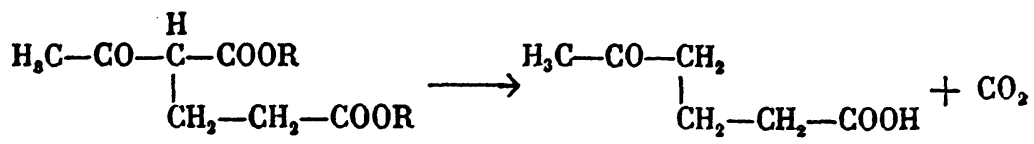

Sie ließ sich der wässerigen Lösung der Verseifungsprodukte mit Hilfe von Chloroform entziehen, während die Oxysäuren und deren Lactone aus der Lösung erst durch Äther weggenommen wurden. Der Rückstand der ätherischen Lösung, der, wie erwähnt, gewöhnlich amorph erschien, zeigte nun bei einem Versuch nach längerer Aufbewahrung einige Krystalle, die mechanisch entfernt werden konnten. Durch Impfen mit denselben gelang es dann bei zwei weiteren Versuchen, zu denen jedesmal $9 \mathrm{~g}$ Acetylglutarsäureester verwendet worden waren, so viel krystallisiertes Material zu erhalten, daß eine analytische Untersuchung ermöglicht wurde. Diese ergab das Vorliegen einer racemischen Oxysäure $\mathrm{C}_{8} \mathrm{H}_{12} \mathrm{O}_{7}+\mathrm{H}_{2} \mathrm{O}$, die als die eine Form der theoretisch möglichen Konfigurationen einer $\beta-0 x y-\beta r \epsilon-$ pentantricarbonsäure angesprochen werden muß, während der früher beschriebene amorphe Stoff beide Formen enthalten haben dürfte, da die aus ihm hergestellten Baryumsalze ganz andere Löslichkeitsverhältnisse aufwiesen, als sie jetzt an dem aus der krystallisierten Säure hergestellten Baryumsalz beobachtet wurden.

Als ein neues Resultat kann ferner mitgeteilt werden, daß beim Erhitzen der Oxysäuren unter gewöhnlichem Druck ebenfalls Hämatinsäure entsteht, und bei der Destillation unter vermindertem Druck wurde neben Methyläthylmaleinsäureanhydrid und Hämatinsäure auch eine Säure beobachtet, die nach Entfernung des Anhydrids, das mit Wasserdämpfen flüchtig ist, und nach Extraktion der Hämatinsäure mittels Chloroform in der wässerigen Lösung zurückblieb und sich dieser durch Äther entziehen ließ. Ihr kommt die empirische Formel $\mathrm{C}_{7} \mathrm{H}_{10} \mathrm{O}_{4} \mathrm{zu}$, beim Erhitzen bis zum Schmelzpunkt tritt unter Gasentwicklung Zersetzung ein. Die gleiche Säure wurde 
dann bei der Destillation des Produktes aufgefunden, das aus Hämatinsäure beim Kochen mit Laugen entsteht und als eine $r$-Lactonsäure in Beziehung zum Carboxyl $€$ nach der Analyse angesprochen werden konnte, während die beschriebene synthetisch gewonnene Oxysäure ein $\delta$-Lacton geben kann:<smiles>CCCCC(O)(C(=O)O)C(C)C(=O)O</smiles>

Nach diesen Beobachtungen war es wahrscheinlich, daB auch die Hämatinsäure bei der Destillation die neue Säure liefern würde, die nach ihren Eigenschaften als ein Derivat einer Itaconsäure angesprochen werden muß, und der Versuch hat dieser Erwartung entsprochen. Auch hier handelte es sich aber stets um so kleine Mengen, daß eine Feststellung der Konstitution bisher nicht erfolgen konnte. Allem Anschein nach ist sie identisch mit der von $W$. Küster beschriebenen Säure $\mathrm{C}_{7} \mathrm{H}_{10} \mathrm{O}_{4}$, die neben Methyläthylmaleinsäureanhydrid bei der Verseifung des Imids entstand, das durch Erhitzen von Hämatinsäure mit alkoholischem Ammoniak bei $130^{\circ}$ unter Abspaltung von Kohlendioxyd hervorgegangen war. ${ }^{1}$ ) Wenigstens zeigte ein Gemisch der beiden Säuren unter gleichen Bedingungen den gleichen Schmelz- resp. Zersetzungspunkt wie die einzelnen Säuren. ${ }^{2}$ )

Da nun die von Fr. Fichter und E. Rudin ${ }^{3}$ ) aus Methyläthylmaleinsäure durch Kochen mit Natronlauge erhaltenen Säuren andere Schmelzpunkte aufweisen und von Fichter und Schlaepfer ${ }^{4}$ ) als Itaconsäuren angesprochen werden, die

1) Liebigs Annalen der Chemie, Bd. 315, S. 216 (1900).

2) Der Schmelz- resp. Zersetzungspunkt wird hier bei $175-176^{\circ}$ angegeben, bei schnellerem Erhitzen liegt er bei $180^{\circ}$; den gleichen Zersetzungspunkt zeigte ein von Herrn Weller dargestelltes Präparat und. die Mischprobe.

3) Ber. d. d. chem. Ges., Bd. 37, S. 1615 (1904).

4) Ber. d. d. chem. Ges., Bd. 39, S. 1535 (1906): 
bei $202^{\circ}$ schmelzende als $\alpha-\beta$-Dimethylitaconsäure, die bei $150^{\circ}$ schmelzende als $\beta$-Äthylitaconsäure, ${ }^{1}$ )<smiles>CC=C(C(=O)O)C(C)C(=O)O</smiles>

und<smiles>C=C(CC)C(CC)C(=O)O</smiles>

und unsere Säure sowohl aus dem $\delta$ - wie aus dem $r$-Lacton entsteht, könnte man versucht sein, an eine Aticonsäure $2 u$ denken:
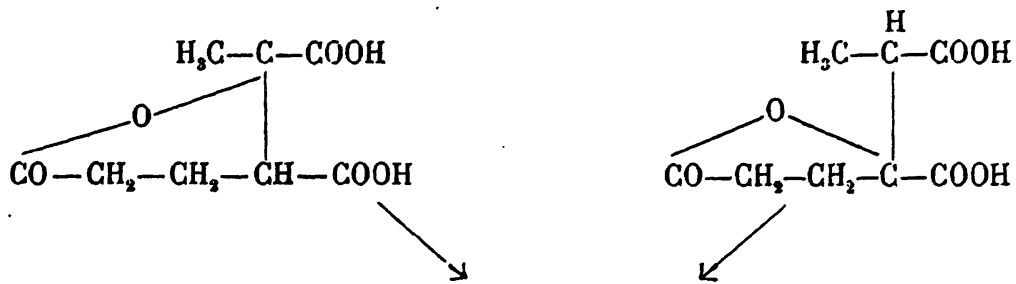<smiles>C=CC(C(=O)O)C(C)C(=O)O</smiles>

doch schließen die Eigenschaften der Säure die Zugehörigkeit zu dieser Klasse aus.2) Danach bleibt die Möglichkeit offen, daß die bei $180^{\circ}$ schmelzende Säure die geometrisch isomere Form zu der von Fichter beschriebenen $\alpha$ - $\beta$-Dimethylitaconsäure ${ }^{3}$ ) vorstellt, welche übrigens auch ein asymmetrisches Kohlenstoffatom aufweist, so.daß von den optisch aktiven Säuren sogar vier verschiedene Konfigurationen theoretisch

1) Nach der von Fittig eingeführten Nomenklatur.

2) Die von Fittig (Liebigs Ann. der Chemie, Bd. 304, S. 211 und 313 [1899]) beschriebenen Aticonsäurederivate, Dimethyl- und Isobutylaticonsäure, schmelzen ohne Zersetzung.

3) Bemerkenswert ist, daß die Abspaltung des Carboxyls erfolgen muß, das die Lactonbindung eingeht, weil dies bei den Paraconsäuren nicht der Fall ist. Nach Fichter und Rudin liefert z. B. $\alpha$-r-Dimethylparaconsäure bei der Destillation $\alpha$-Methyl- $\beta-\gamma-$ pentensäure:

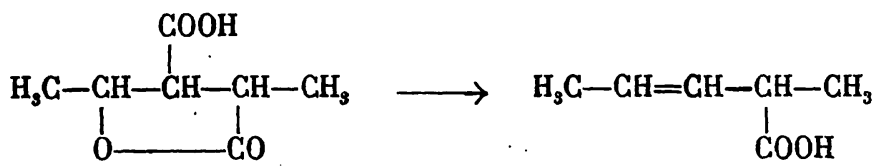


möglich sind.1) Da sich nun ferner diese Säure aus der Hämatinsäure beim Erhitzen bildet, wäre es nicht ausgeschlossen, daß letztere dabei eine teilweise Umlagerung erleidet derart, daß sich das Lacton der $\gamma$ - oder auch das der $\delta$-0xysäure bildet:<smiles>CCCCC(C)=C(C)C(=O)OC</smiles><smiles>CC1OOOC12CCC(=O)O2</smiles>

Und diese Umwandlung könnte sich auch schon beim Erhitzen der Hämatinsäure mit alkoholischem Ammoniak unter Druck vollzogen haben, so daß das hierbei unter Abspaltung von Kohlendioxyd gebildete Imid, das zunächst d. h. im rohen Zustande immer einen unscharfen Schmelzpunkt aufwies, bereits das Imid der Itaconsäure enthalten hat, das dann bei der Verseifung die kleine Menge der Itaconsäure lieferte. Zurzeit liegt allerdings kein anderer Beweis für diese Anschauung, vor, als die Beobachtung Fichters, wonach sich bei der Einwirkung von Natronlauge auf Methyläthylmaleinsäure andere Säuren bilden, und unsere Säure demnach nicht aus dem Methyläthylmaleinimid erst bei der Verseifung mit Barytwasser entstanden sein kann. Bei diesem ProzeB erhielt nun der eine von uns neben den Baryumsalzen der Methyläthylmaleinsäure und der Itaconsäure auch eine hornartige Masse als leicht löslichen Rückstand, ${ }^{2}$ ) und es ist nicht unwahrscheinlich, daß in diesen Baryumsalzen solche von Oxysäuren vorliegen, die sich durch Anlagerung von Wasser an die ungesättigte Säure gebildet haben. Erleidet doch die Hämatinsäure, wie schon erwähnt, die gleiche Umwandlung unter ähnlichen Bedingungen, allerdings erst beim Kochen mit starken Laugen, nicht durch Barytwasser, wie ein besonderer Versuch erwiesen hat. Dieses Verhalten bietet daher noch keine Erklärung für.

1) Unsere Säure konnte der kleinen Menge wegen, die wir in Händen haben, nicht auf optische Aktivität untersucht werden. Es ist auch wenig wahrscheinlich, daß sie solche aufweisen wird.

2) Liebigs Annalen, Bd. 345, S. 212 (1905). 
die öfters beobachteten schlechten Ausbeuten an reiner Hämatinsäure, wenn zur Reinigung des bei der Oxydation des Hämatins gebildeten rohen Gemisches von Hämatinsäureimid und -anhydrid die Verseifung mit Barytwasser angewendet worden war, wobei neben dem unlöslichen Baryumsalz der Hämatinsäure auch leicht in Wasser lösliche Baryumsalze auftreten. Da nun letztere sich von 0xysäuren ableiten dürften, denn es gelang wenigstens, die aus ihnen hergestellten Säuren durch Erhitzen in Hämatinsäure überzuführen, d. h. unter gleichen Bedingungen wie die synthetische $\delta$-Oxysäure und die $r$-Lactonsäure, so müssen diese Oxysäuren gleichfalls aus der Oxydation des Hämatins hervorgehen. Wahrscheinlich haben sie sich unter der Einwirkung der bei diesem Prozesse verwendeten Schwefelsäure aus der Hämatinsäure gebildet. ${ }^{1}$ ) Dafür spricht die oft mangelhafte Ausbeute an reiner Hämatinsäure bei der Verseifung des Hämatinsäureimids durch Schwefelsäure ${ }^{2}$ ) und ferner die neuerdings gemachte Beobachtung, da $B$ viel leicht lösliches Baryumsalz entsteht, wenn die Oxydation des Hämatins in schwefelsaurer Lösung durch Chromsäure vorgenommen wird.

Doch wäre auch noch an eine andere Möglichkeit für das Entstehen von Oxysäure zu denken, deren Auftreten die Ausbeuten an Hämatinsäuren herabdrücken muß, wie dies von uns namentlich festgestellt werden konnte, wenn alte Häminpräparate der Oxydation unterzogen worden waren. Wenn nämlich die Hämatinsäure eine Lactonsäure durch Umlagerung geben kann, so ist eine Übertragung der Anschauung auf das Hämatin selbst in Betracht zu ziehen, d. h. es kann für die carboxylierten Pyrrolkerne im Hämatin mit einer Umlagerungsmöglichkeit im folgenden Sinne gerechnet werden:

1) Am einfachsten lassen sich die ungesättigten Säuren durch verdünnte Schwefelsäure in Lactone überiühren, indem man sie mit einem Gemisch gleicher Volumina konzentrierter Schwefelsäure und Wasser kurze Zeit erwärmt. Fittig, Ber. d. d. chem. Ges., Bd. 16, S. 373 (1883), St o b be und Vieweg, Ber., Bd. 35, S. 1734 (1902).

2) Mezger, Dissertation (Tübingen 1902), S. 12/14; Lacour, Dissertation (Würzburg 1907), S. 37. 


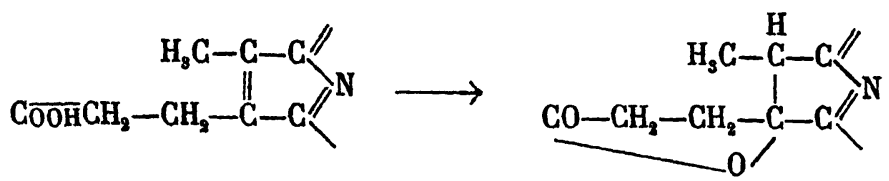

Eine solche Umlagerung, wie sie von W. Küster in ähnlicher Art auch schon für das Bilirubin ${ }^{1}$ ) angenommen worden ist, könnte dann vielleicht auch für die Unlöslichkeit einiger Monoalkylester des Hämins in Alkalien als Erklärung herangezogen werden.

\section{Experimenteller Teil.}

\section{Die Darstellung des Acetylglutarsäureesters}

erfolgte nach der von Conrad und Limpach ${ }^{2}$ ) gegebenen Vorschrift für die Gewinnung von Acetsuccinsäureester.

2,3 $\mathrm{g}$ Natrium wurden in $32 \mathrm{~g}$ absoluten Alkohol gelöst, nach dem Abkühlen erfolgte der Zusatz von $14 \mathrm{~g}$ Acetessigester $^{3}$ ) und sofort darauf von 22,8 g $\beta$-Jodpropionsäureester, $\left.{ }^{4}\right)$ wonach Erwärmung und Trübung erfolgte. Nach zweistündigem Erwärmen auf dem Wasserbade am Rückflußkühler wurde der Alkohol abdestilliert, das abgeschiedene Jodnatrium in Wasser, die organischen Stoffe in Äther gelöst und die ätherische Lösung mit kleinen Mengen Wassers geschüttelt, bis die Reaktion auf Jodionen verschwunden war. Aus der getrockneten ätherischen Lösung wurde der Äther abdestilliert und der Rückstand bei vermindertem Druck fraktioniert: Die bei 22-25 mm bei $169-171^{\circ}$ siedende Fraktion - ein farbloses, schwach riechendes Öl - stellte den Acetylgutarsäureester vor.

$$
\begin{array}{r}
0,1970 \mathrm{~g} \text { Substanz : } 0,4137 \mathrm{CO}_{2} \text { und } 0,1315 \mathrm{H}_{8} \mathrm{O} . \\
\mathrm{C}_{11} \mathrm{H}_{18} \mathrm{O}_{5} \text {. Ber.: } 57,4 \% \mathrm{C} \text { und } 7,8 \% \mathrm{H} \\
\text { Gef.: } 57,26 \% \text {, } 7,42 \% \mathrm{H} .
\end{array}
$$

1) Diese Zeitschrift, Bd. 99, S. 98 (1917).

2) Liebigs Ann: der Chemie, Bd. 192, S. 157 (1878).

3) Berechnet sind $13 \mathrm{~g}$.

4) Der von Kahlbaum bezogene Ester wurde nach den Angaben von J. Wislicenus (Ann., Bd. 192, S. 128) durch Schütteln mit verd. Natronlauge gereinigt und über geglühter Pottasche getrocknet. 


\section{Die Anlagerung von Blausäure an den Acetylglutar-} säureester

geschah in Anlehnung an die für die Darstellung des Nitrils des Methyläthyläpfelsäureesters von $\mathrm{K}$. Haas ausgearbeitete Vorschrift') in der Weise, daß die Lösung von $9 \mathrm{~g}$ des Esters in $27 \mathrm{~g}$ Äther mit 7,5 $\mathrm{g}$ feinst gepulvertem reinen Cyankalium ${ }^{2}$ ) versetzt und das gekühlte Gemisch mit der berechneten Menge Salzsäure $(10,5 \mathrm{~g}$ vom spez. Gew. 1,19$)$ in kleinen Portionen versetzt wurde. Beim Zusatz des Cyankaliums zur ätherischen Lösung des Esters trat immer eine Gelbfärbung des Salzes auf, die auf Zusatz der Salzsäure sofort verschwand. Nach dreitägiger Einwirkung waren die ätherischen Lösungen, welche das Anlagerungsprodukt enthielten, immer nur schwach gelb getärbt, die Abscheidung einer wässerigen Schicht wurde nicht beobachtet. Die ätherische Lösung wurde dann durch Watle filtriert, das feuchte Salz wiederholt mit Äther ausgewaschen, der Äther abdestilliert und der Rückstand zur Vertreibung anhaftender Blausäure unter Durchleiten von Luft im Wasserbade erwärmt. Es hinterblieb dann ein bräunlich gefärbtes Öl von eigenartigem, nicht gerade unangenehmem Geruch.

\section{Die Verseifung des Cyanwasserstoffanlagerungs- produktes}

wurde durch vierzehnstündiges Erhitzen mit $70 \mathrm{~g} 25 \%$ iger Salzsäure im Wasserbade am Rückflußkühler bewirkt. Ob eine kürzere Behandlung angängig ist, wurde nicht erprobt, doch sei bemerkt, daß von dem anfangs auf der Salzsäure schwimmenden Öl schon nach halbstündigem Erwärmen nur noch Spuren vorhanden waren. Die braungelbe Flüssigkeit wurde nunmehr in einem Extraktionsapparat mit Äther ausgezogen, was trotz tagelanger Behandlung nicht vollständig gelang, da der nach dem. Verdampfen der salzsauren Lösung hinterbleibende

1) Liebigs Annalen der Chemie; Bd. 345, S. 12 (1905).

2) Diese Menge stellt das Dreifache der berechneten vor, welcher Überschuß sich für Erlangung guter Ausbeuten als höchst zweckmäßig erwiesen hat. Besonderer Wert ist auch darauf zu legen, daß das Cyankalium aufs feinste gepulvert ist. 
Rückstand an Äther noch Substanz abgab. Aus der ätherischen Lösung wurde dann das Lösungsmittel abdestilliert, der Rückstand in Wasser gelöst, und diese Lösung mit Chloroform extrahiert, wodurch ein Nebenprodukt beseitigt wurde. Es erwies sich nach allen Eigenschaften als Acetylbuttersäure, durch Verseifung derjenigen Teile des Acetylglutarsäureesters entstanden, an die eine Anlagerung von Blausäure nicht erfolgt war. Ihre Reinigung erfolgte nach Abdestillation des Chloroforms durch Aufnahme des Rückstands in Wasser und Extraktion mit Äther. Der Schmelzpunkt des so gewonnenen Hydrats der Acetbuttersäure lag bei $35-36^{\circ}$.

Bei diesen Versuchen wurde auch festgestellt, daß dieses Hydrat mit Wasserdämpfen nur in sehr geringem Grade flüchtig ist, während die wasserfreie Acetbuttersäure sich schon bei Zimmertemperatur ein wenig, bei Wasserbadtemperatur erheblich verflüchtigt.

Die von der Acetylbuttersäure befreite Lösung mußte das gewünschte Produkt, die 0xysäure<smiles>CC(=O)C(CCC(=O)O)C(C(=O)O)C(=O)O</smiles>

enthalten, da eine Probe des Rückstands beim Erhitzen den Geruch nach Methyläthylmaleinsäureanhydrid deutlich gab. Bei einem zweiten Versuch wurden aus $0,455 \mathrm{~g}$ des Rückstandes 0,3 g Destillat erhalten, während 0,04 g eines braunen, harzigen Stoffes im Entwicklungsgefäß blieben. Das Destillat wurde nunmehr mit Wasserdämpfen behandelt, wobei eine Trennung erreicht wurde.

a) Das Wasserdampfdestillat, das Öltropfen enthielt, verbrauchte, heiß mit Phenolphthalein als Indikator titriert, $12,6 \mathrm{ccm} 1 / 5-n$-Barytwasser. Beim Einkochen der Baryumsalzlösung auf $28 \mathrm{~g}$ trat ziemlich reichliche Abscheidung von Krystallen ein, die unter dem Mikroskop als Geschiebe von Blättchen erkannt wurden. Beim Erkalten lösten sie sich. allmählich wieder auf. Danach lag das Baryumsalz der Methyläthylmaleinsäure vor, deren Menge sich nach dem Barytverbrauch $\mathrm{zu} 0,1764 \mathrm{~g}$ berechnet. 
b) Die rückständige Flüssigkeit hinterließ beim Eindampfen 0,02 g einer schwach gelblich gefärbten krystallinischen Substanz, die bei $150-160^{\circ}$ schmolz und bei weiterem Erhitzen Gasbläschen entwickelte. Hämatinsäure war somit ausgeschlossen. ${ }^{1}$ )

Ferner wurde festgestellt, daß die Destillation unter Entwicklung von Kohlendioxyd erfolgte, die bei $160-180^{\circ}$ sehr schwach war und erst beim Erhitzen bis $230^{\circ}$ stark einsetzte. Bei dieser Temperatur des Bades und Verminderung des Druckes auf $10 \mathrm{~mm}$ erfolgte dann auch die vollständige Destillation der beiden beschriebenen Säuren. War somit auch jeder Zweifel behoben, daß die gesuchte Oxysäure durch die Synthese erhalten worden war, so erwiesen doch die Titrationen bestimmter Mengen des sirupartigen Stoffes mit Barytwasser bei Zimmertemperatur und beim Kochen, daß es sich um ein Gemisch von Oxysäuren und deren Lactonen handelte, denn, wenn die Synthese den gewünschten Verlauf genommen hatte, mußte ein Gemisch von zwei racemischen Oxysäuren vorliegen, da die $\beta-0 x y-\beta-\gamma-\epsilon-$ pentantricarbonsäure $z$ wei asymmetrische Kohlenstoffatome aufweist. Nun wurde noch festgestellt, daß die Aufspaltung des Lactonrings durch Barytwasser bereits bei Zimmertemperatur erfolgte, daß längeres Kochen ohne Einfluß auf das Titrationsergebnis war und daß eine Vermehrung der Lactonbildung durch Erhitzen mit verdünnten Säuren nicht zu erreichen war, die immer nur in geringem Grade eingetreten sein konnte.

a) Je 0,2719 g des Sirups verbrauchten: bei Zimmertemperatur 16,58, in der Hitze 16,43, nachdem 10, 20 und 30 Minuten mit der Barytlösung gekocht worden war, 16,8, 16,8 und $16,75 \mathrm{ccm} 1 / 5-\mathrm{n}-\mathrm{Ba}(\mathrm{OH})_{2}$, während sich für eine dreibasische Säure $\mathrm{C}_{8} \mathrm{H}_{12} \mathrm{O}_{7} 18,54 \mathrm{ccm}$ berechnen.

b) 0,2638 $\mathrm{g}$ Sirup aus einem anderm Versuch verbrauchten zur Neutralisation 15,95 ccm $1 / 5-\mathrm{n}-\mathrm{Ba}(\mathrm{OH})_{2}$. Nach halbstündigem Kochen mit $20 \mathrm{ccm} 1 / 5-\mathrm{n}-\mathrm{H}_{2} \mathrm{SO}_{4}$ verbrauchte eine Probe von gleichem Gewicht $35,8-20=15,8 \mathrm{~cm} 1 / 5-\mathrm{n}-\mathrm{Ba}(\mathrm{OH})_{2}$ bis

1) Die Säure wurde dann später als Itakonsäurederivat erkannt, vgl. Teil VI. 
zur Neutralisation' bei Zimmertemperatur, zur Verseifung in der Hitze dann noch $1,1 \mathrm{ccm}$.

Die Herstellung eines Baryumsalzes aus den mit Chloroform extrahierten Lösungen des Rohproduktes, welche das Gemisch der Oxysäure mit ihren Lactonen enthielten, erfolgte durch Zusatz von Barytwasser im Überschuß, Entfernung des letzteren durch Einleiten von Kohlendioxyd, Aufkochen und Filtrieren vom Baryumcarbonat, wonach das Filtrat verdampft wurde. Das so erhaltene amorphe Baryumsalz löste sich in etwa der zehnfachen Menge siedenden Wassers und enthielt nach dem Trocknen bei $100^{\circ} 46,27 \% \mathrm{Ba}$, während sich für das Salz $\left(\mathrm{C}_{8} \mathrm{H}_{9} \mathrm{O}_{7}\right)_{2} \mathrm{Ba}_{3} 48,64 \% \mathrm{Ba}$ berechnen. Es mußte also schon während der Darstellung wieder zur Bildung von Lacton gekommen sein. Es wurde deshalb in der Erwartung, daß die Lactonsäure zuerst durch Salzsäure in Freiheit gesetzt wird, zur fractionierten Zerlegung des Baryumsalzes geschritten. Doch zeigte sich, daß Äther aus der mit Salzsäure nur schwach übersättigten Lösung des Baryumsalzes (angewendet 6,2 g Salz und $9 \mathrm{~g}$ Salzsäure, ber. $6,1 \mathrm{~g} 25 \%$ ige $\mathrm{HCl}$ zur Bindung des Baryums) so gut wie keine organische Säure wegnahm. Erst auf Zusatz von $40 \mathrm{~g} \mathrm{HCl}$ wurden 1,3, nach weiterem Zusatz von $20 \mathrm{~g} \mathrm{1,4} \mathrm{g}$ Ätherextrakt erhalten. Die Titration mit Barytwasser zeigte dann, daß die $1,3 \mathrm{~g}$ etwa $1 / 3$, die $1,4 \mathrm{~g}$ etwa 1/4 Lactonsäure enthalten mußte.

Es war aber durch diese Behandlung insofern eine Änderung in der Zusammensetzung des Gemisches der Säuren mit ihren Lactonen eingetreten, als die jetzt erhaltenen Baryumsalze sich bereits in kaltem Wasser spielend lösten. Es gelang, aus der Fraktion von 1,3 g eine $28 \%$, aus der von $1,4 \mathrm{~g}$ eine $44 \%$ Baryumsalz enthaltende Lösung herzustellen. Diese Lösungen erstarrten beim Einstellen in kochendes Wasser zu einer Gallerte, die beim Erkalten wieder flüssig wurde und in lauem Wasser sich vollständig und klar wieder löste. Eine 19\% ige Lösung gab beim Erhitzen noch eine erhebliche, eine 14\% ige nur noch eine geringe Abscheidung. Die konzentrierten Lösungen zeigten gegen Lackmus alkalische Reaktion, sehr verdünnte eine amphotere. Kohlendioxyd, in 
eine $90^{\circ}$ heiße $7 \%$ ige Lösung eingeleitet, bewirkte nur eine schwache Trübung.

Das aus einer 4\% igen Lösung dieser Baryumsalze hergestellte Silbersalz zeigte nach dem Trocknen bei $90^{\circ}$ die der Formel $\mathrm{C}_{8} \mathrm{H}_{9} \mathrm{Ag}_{3} \mathrm{O}_{7}$ etwa entsprechenden Werte I; doch ist auch die Formulierung mit $1 / 2 \mathrm{H}_{2} \mathrm{O}$ in Betracht zu ziehen. Wenigstens stimmten die bei einer zweiten Analyse gefundenen Werte besser auf die letztere. Hier war das Silbersalz direkt aus dem mit Ammoniak neutralisierten Gemisch der Oxysäuren und ihrer Lactone durch Silbernitrat als voluminöser, weißer, in kaltem Wasser sehr schwer löslicher Niederschlag gefällt worden, der nach dem Trocknen im Vakuum sich ein wenig verfärbt hatte:

II. 0,2740 g Substanz : $0,1730 \mathrm{CO}_{2}, 0,0537 \mathrm{H}_{2} \mathrm{O}$ und $0,1607 \mathrm{Ag}$ $\mathrm{C}_{8} \mathrm{H}_{9} \mathrm{Ag}_{9} \mathrm{O}_{7}+1 / 2 \mathrm{H}_{2} \mathrm{O}$. Ber.: $17,46 \% \mathrm{C} \quad 1,82 \% \mathrm{H} \quad 58,91 \% \mathrm{Ag}$

I. ${ }^{1)}$ Gef.: $17,57 \% \quad 1,77 \% \quad 59,11 \%$ II. $\quad 17,22 \% \quad 2,18 \% \quad 58,65 \%$.

Die Gewinnung der Hämatinsäure aus dem Gemisch der Oxysäuren mit ihren Lactonen durch Erhitzen unter vermindertem Druck ist bereits in den «Berichten» beschrieben. Wir können nunmehr ergänzend mitteilen, daß die zum Gelingen dieses Vorgangs nötige Wasserabspaltung ohne Zersetzung unter Entwicklung von Kohlendioxyd sich auch unter gewöhnlichem Druck vollziehen läßt. Es wurden hierbei 0,3705 g des Sirups in einem Paraffinbade erhitzt, dessen Temperatur binnen 36 Minuten von $175^{\circ}$ auf $181^{\circ}$ stieg. Das Gewicht des Rückstandes betrug dann 0,3424 g. Die Lösung desselben in $10 \mathrm{~g}$ Wasser gab bei dreimaligem Ausschütteln mit je $15 \mathrm{ccm}$ Chloroform 0,213 g Extrakt, das fast farblos war und sofort nach dem Abkühlen in den bekannten Formen der Hämatinsäure krystallisierte. Beim Eindampfen der mit Chloroform ausgeschüttelten Flüssigkeit blieben 0,104 g Rückstand. Durch Erhitzen desselben im Paraffinbade und einmaliges Auskochen des Produktes mit Chloroform wurden noch 0,072 g Extrakt gewonnen, das braun gefärbt war und erst nach längerer Zeit krystallisierte. Doch lag auch hier

1) Vgl. Ber. d. d. chem. Ges., Bd. 47, S. 534 (1904). 
Hämatinsäure vor, so daß die Ausbeute an derselben über

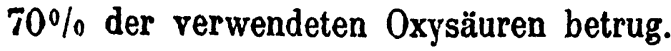

IV. Die rac. $\beta-0 x y$ pentan- $\beta-\gamma-\epsilon-$ tricarbonsäure.

Bei einem unserer Versuche zeigten sich in dem Rückstand des ätherischen Auszugs der wässerigen Lösung, die nach Verseifung des Blausäureanlagerungsproduktes an den Acetylglutarsäureester mittels Salzsäure, Extraktion dieser Lösung mit Äther und Aufnahme des Rückstands in Wasser erhalten und zur Entfernung der Acetbuttersäure mit Chloroform extrahiert worden war, nach wochenlangem Stehen einige Krystalle, die mechanisch entfernt und durch Betupfen mit Äther von der anhängenden Mutterlauge befreit werden konnten. Durch Impfen mit diesen Krystallen gelang es dann, bei einigen weiteren Versuchen eine teilweise Krystallisation des sirupösen Ätherrückstandes zu bewirken. Die Trennung von diesem erfolgte durch Äther, in welchem die Krystalle schwerer löslich waren. Aus zwei Versuchen mil je 9,5 Acetglutarsäureester wurden hierbei 3,6 $\mathrm{g}$ einer farblosen Säure erhalten, die zunächst bei $103-106^{\circ} \mathrm{schmolz}$; durch zweimaliges Umkrystallisieren aus heißem Wasser verblieben $0,8 \mathrm{~g}$ krystallisierte Säure, $\left.{ }^{1}\right)$ die den Schmelzpunkt $108-110^{\circ}$ aufwiesen. Diese, nicht hygroskopische Säure zeigte beim Trocknen im Exsikkator keine Abnahme und erwies sich als das Hydrat der Oxysäure $\mathrm{C}_{8} \mathrm{H}_{1 \mathrm{~g}} \mathrm{O}_{7}$.

$$
\begin{gathered}
\text { 0,1993 Substanz : } 0,2950 \mathrm{CO}_{2} \text { und } 0,1059 \mathrm{H}_{2} \mathrm{O} . \\
\mathrm{C}_{8} \mathrm{H}_{12} \mathrm{O}_{7}+\mathrm{H}_{2} \mathrm{O} \text {. Ber.: } 40,34 \% \mathrm{C} \text { und } 5,88 \% \mathrm{H} . \\
\text { Gef.: } 40,37 \% \quad 5,9 \% .
\end{gathered}
$$

Titration. $0,3082 \mathrm{~g}$ Substanz verbrauchten zur Neutralisation 19,4 1/5-n- $\mathrm{Ba}(\mathrm{OH})_{2}$ (Phenolphthalein), berechnet für $\mathrm{C}_{3} \mathrm{H}_{12} \mathrm{O}_{7} \mathrm{H}_{2} \mathrm{O}$, dreibasisch $19,4 \mathrm{ccm}$.

Die sodaalkalische Lösung der Säure erwies sich gegen Kaliumpermanganat ziemlich beständig, d. h. die Entfärbung

2) Aus den Mutterlaugen wurde noch $1 \mathrm{~g}$ unscharf zwischen 104-110 schmelzende Säure gewonnen, dann hinterblieb eine zähe Masse, die nicht zum Krystallisieren zu bringen war. 
trat erst nach einstündiger Einwirkung ein. Bei längerem Erhitzen auf $90^{\circ}$ verliert die krystallisierte Säure nicht nur das Krystallwasser, sondern außerdem noch fast zwei Molekeln Wasser, so daß es also zur Lacton- und zur Anhydridbildung kommt.

$0,5025 \mathrm{~g}$ Subst. verloren bei 1 tägigem Erhitzen auf $90^{\circ} 0,0586 \mathrm{~g}=11,66^{\circ} \%$ $0,5025,>, 2, \quad>\quad 90^{\circ} 0,0703,=13,99 \%$ 0,5025, , , , > , , $90^{\circ} 0,0796$, $=15,84 \%$ $0,5025,>, 5, \quad, \quad, 90^{\circ} 0,0814>=16,2 \%$ 0,5025, , , , , , $90^{\circ} 0,0869>=17,3 \%$

Bei einem zweiten Versuch wurde ein Gewichtsverlust bis $20,4 \%$ beobachtet. Berechnet für $1 \mathrm{H}_{2} \mathrm{O}: 7,56 \%, 2 \mathrm{H}_{2} \mathrm{O}$ $15,13 \%, 3 \mathrm{H}_{2} \mathrm{O} 22,69 \%$.

Der Schmelzpunkt der erhitzten Säure lag nach Verlust von $15,8 \%$ bei $160-165^{\circ}$, nach Verlust von $20,4 \%$ bei 163-164. Aus der Luft nahm die erhitzte Säure nur Spuren von Wasser auf, beim Lösen in Wasser wurde das Hydrat $\mathrm{C}_{8} \mathrm{H}_{12} \mathrm{O}_{7}+\mathrm{H}_{2} \mathrm{O}$ wieder erhalten. Der Schmelzpunkt war zwar unscharf geworden, er lag bei $103-109,5^{\circ}$, die Titration gab aber das berechnete Resultat: 0,1827 Substanz verbrauchten $11,35 \mathrm{ccm} 1 / 5-\mathrm{n}-\mathrm{Ba}(\mathrm{OH})_{2}$, statt $11,5 \mathrm{ccm}$.

Das Baryumsalz der krystallisierten Säure zeigte bemerkenswerterweise ganz andere Eigenschaften, wie das aus der sirupösen Säure gewonnene, bei Zimmertemperatur löste es sich schwer - die Lösung enthielt nur etwas über $2 \%$ des Salzes -, in siedendem Wasser löste es sich viel leichter und krystallisierte aus der heiß gesättigten Lösung sehr langsam in feinen Nadeln aus. Genau so verhielt sich auch das Baryumsalz, das aus der auf $90^{\circ}$ erhitzten Säure nach dem Wiederlösen in Wasser dargestellt worden war.

Nach diesen Befunden muß angenommen werden, daß die krystallisierte Säure die eine Form der beiden theoretisch

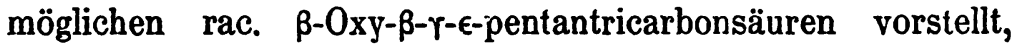
während die andere Form in den sirupösen Mutterlaugen zurückblieb. Der Versuch, die krystallisierte Säure in Hämatinsäure umzuwandeln, konnte bisher aus Mangel an Material nicht ausgeführt werden; gelingt er, woran übrigens nach den 
fast quantitativen Ausbeuten bei Verwendung des Gemisches nicht zu zweifeln ist, so muß nach den beim Erhitzen auf $90^{\circ}$ gemachten Beobachtungen geschlossen werden, daß beim Erhitzen bis $180^{\circ}$ lediglich eine Umwandlung durch Wanderung eines Wasserstoffatoms eintritt, da sich ja bereits bei $90^{\circ}$ das Anhydrid der Lactonsäure gebildet hat:<smiles>CC(O)(C(=O)O)C(CCC(=O)O)C(=O)O</smiles><smiles>CCCCCCC</smiles><smiles>COCCC1OOOC1(C)C</smiles><smiles>CC1=C(C)C(CCC(=O)O)COO1</smiles>

\section{Bildung einer $r$-Lactonsäure aus der Hämatin- säure.}

Beim Kochen der Hämatinsäure mit starken Langen findet eine teilweise Umwandlung derselben statt, wie in den *Berichten» bereits kurz erwähnt wurde. Die Versuche wurden in Anlehnung an die von Fittig ${ }^{1}$ ) über die Umlagerung ungesättigter Säuren unternommen, und es wurde namentlich mit der Möglichkeit gerechnet, die fumaroide Form der Hämatinsäure zu gewinnen. Daneben konnte an die Bildung strukturisomerer Säuren gedacht werden, die sich von der Itakonsäure ableiten. Das Entstehen einer Oxysäure resp. Lactonsäure wurde zunächst nicht in Betracht gezogen; allerdings batte schon Fittig, da er bei seinen Umlagerungsversuchen einen Verlust von durchschnittlich $10 \%$ beobachtete, die Vermutung geäußert, $\left.{ }^{2}\right)$ daß wahrscheinlich 0xysäure entstanden sei, die sich durch Äther nicht extrahieren lasse, ${ }^{3}$ ) und von van't Hoff war aus Maleinsäure durch längeres Erhitzen mit Natronlauge auf $100^{\circ}$ Äpfelsäure erhalten worden. ${ }^{4}$ )

1) Liebigs Ann. der Chem.; Bd. 304, S. 117.

2) Ibid., Bd. 304, S. 153 (1899).

3) Hierzu sei bemerkt, daB sich Methyläthyläpfelsäure aus stark salzsäurehaltiger Lösung durch Äther extrahieren läbt, andere zweibasische Oxysäuren werden sich wohl ähnlich verhalten.

4) Ber. d. d. chem. Gesellsch., Bd. 18, S. 2713 (1885). 
Die Versuche mit unserer dreibasischen Säure ergaben nun das interessante Resultat, daß sich eine Lactonsäure bildet, daß sich aber ein Gleichgewicht einstellt, wenn sich etwa $72 \%$ der angewandten Menge Hämatinsäure umgewandelt haben. Demnach mußte auch dieser ProzeB umkehrbar sein, was der Versuch bestätigt hat (vgl. Seite 247).

Wie sich aus der Tabelle ergibt, wird dieses Gleichgewicht durch Verwendung $40 \%$ iger Kalilauge in $9-10$ Stunden, bei $33 \%$ iger erst in 22 Stunden erreicht.

\begin{tabular}{|c|c|c|c|c|c|c|c|c|c|c|}
\hline $\begin{array}{l}\text { Nr. } \\
\text { des } \\
\text { Ver- } \\
\text { suchs }\end{array}$ & $\begin{array}{l}\text { Hä- } \\
\text { matin- } \\
\text { säure } \\
\text { g }\end{array}$ & $\begin{array}{c}\text { Menge } \\
\text { der } \\
\text { Kali- } \\
\text { lauge } \\
\text { g }\end{array}$ & $\begin{array}{c}\text { Proz.- } \\
\text { Gehalt } \\
\text { d. Kali- } \\
\text { lauge } \\
\%\end{array}$ & $\begin{array}{c}\text { Dauer } \\
\text { des } \\
\text { Ko- } \\
\text { chens } \\
\text { Std. }\end{array}$ & $\begin{array}{l}\text { Tem- } \\
\text { pe- } \\
\text { ratur }\end{array}$ & $\begin{array}{c}\text { Menge } \\
\text { der } \\
\text { Salz- } \\
\text { säure }\end{array}$ & $\begin{array}{c}\text { Gewicht } \\
\text { des } \\
\text { Äther- } \\
\text { extrakts }\end{array}$ & $\begin{array}{l}\text { In Chl } \\
\text { lös- } \\
\text { lich }\end{array}$ & $\begin{array}{l}\text { orform } \\
\begin{array}{c}\text { unlös- } \\
\text { lich }\end{array}\end{array}$ & $\begin{array}{l}\text { Um- } \\
\text { gewan- } \\
\text { delte } \\
\text { Säure } \\
\text { in } \%\end{array}$ \\
\hline 1 & 5 & 200 & 30 & 9 & $110^{\circ}$ & \multirow{5}{*}{$\begin{array}{c}162 \mathrm{~g} \\
25 \text { pro } \\
\text { zen- } \\
\text { tige }\end{array}$} & 5 & 2,52 & 2,48 & 50 \\
\hline 2 & 5 & 200 & 30 & 22 & $110^{\circ}$ & & 4,98 & 1,37 & 3,61 & 73 \\
\hline 3 & 5 & 150 & 40 & 9 & $110^{\circ}$ & & 4,84 & 1,2 & 3,6 & 72 \\
\hline 4 & 5 & 150 & 33 & 22 & $110^{\circ}$ & & 4,63 & 1,02 & 3,4 & 68 \\
\hline 5 & 8 & 200 & 40 & 10 & $110^{\circ}$ & & 7,68 & 1,84 & 5,8 & 72,5 \\
\hline
\end{tabular}

Die Durchführung des Prozesses geschah nach einigen Vorversuchen in der Weise, daß die alkalische Lösung der Hämatinsäure in einem Kupferkessel am Rückflußkühler gekocht wurde; nach dem Erkalten wurde vorsichtig angesäuert, ${ }^{1}$ ) die Lösung neunmal mit je $200 \mathrm{ccm}$ Äther ausgeschüttelt, der Rückstand der ätherischen Lösung in Wasser gelöst, und diese Lösung durch eintägige Extraktion mit Chloroform von unveränderter Hämatinsäure befreit.

Die Gewinnung krystallisierender Substanz aus den nur in Äther löslichen. Teilen, also dem Umwandlungsprodukt,

1) Der Zusatz der Salzsäure erfolgte allmählich und unter Kühlung, um einer Umlagerung vorzubeugen, da Fittig (Ann., Bd. 304, S. 126) beobachtet hatte, daß sich aus Isopropyl-Itakonsäure Isopropyl-Isoparaconsäure bildet, wenn zu einer heißen Lösung der ersteren in überschüssiger Natronlauge rasch konzentrierte Salzsäure hinzugesetzt wird:<smiles>CC(C)C=C(CC(=O)O)C(=O)O</smiles><smiles>CC1(C)CC(CC(=O)O)C(=O)O1</smiles> 
stieß auf große Schwierigkeiten. Zwar krystallisierte ein kleiner Teil aus der konzentrierten ätherischen Lösung heraus, wenn dieselbe mit etwas Petroläther versetzt, einige Zeit stehen blieb, und diese Krystalle ließen sich von dem zu gleicher Zeit ausgefallenen Sirup durch Behandeln mit geringen Mengen Äther, der nur den letzteren löste, abtrennen. Doch war die Menge sehr gering. Etwas bessere Resultate in bezug auf die Ausbeute lieferte das Versetzen der konzentrierten Lösung der Säure in wasserhaltigem Äther mit wasserfreiem Äther und Wiederholung des Verfahrens, nachdem der Äther zum Teil abdestilliert worden war. So wurden z. B. aus $5 \mathbf{g}$ : $0,75+0,09+0,04=0,88 \mathrm{~g}$ krystallisierte Säure gewonnen; im ganzen waren es schließlich $2 \mathrm{~g}$, die den scharfen Schmelzpunkt $168-169^{\circ}$ aufwiesen. Die Analyse und die Molekulargewichtsbestimmung ${ }^{1}$ ) wies auf die Formel $\mathrm{C}_{8} \mathrm{H}_{10} \mathrm{O}_{6}$, die Titrierung bei Zimmertemperatur und in der Hitze ergab das Vorliegen einer zweibasischen Lactonsäure. In ihr muß das Lacton einer der racemischen Formen einer $\gamma-0 x y-\beta-\gamma-\epsilon$-pentantricarbonsäure:<smiles>CC(C(=O)O)C1(C(=O)O)CCOCC1</smiles>

vorliegen, da die $\delta$-0xysäure andere Eigenschaften besitzt, z. B., wie erwähnt, viel schwerer ein Lacton gibt. Auch ließ sich das Baryumsalz der $r$-Oxysäure, das in kaltem Wasser $\mathrm{zu} 2,1 \%$ löslich ist $[4,0565 \mathrm{~g}$ der Lösung hinterließen $0,0845 \mathrm{~g}$ Rückstand] aus heißem Wasser leicht umkrystallisieren.2)

Die bei $169^{\circ}$ schmelzende Lactonsäure krystallisiert aus Wasser in Tafeln von quadratischem Umriß, die nach Messungen von Herrn Geh. Rat Prof. Dr. Wülffing wahrscheinlich

1) Vgl. Ber. d. d. chem. Ges., Bd. 47, Bd. 532 (1914).

2) Die sirupöse Säure gibt ein Baryumsalz, das sich bei Zimmertemperatur zu $20 \%$ löst, beim Erhitzen der Lösung tritt eine Abscheidung auf. In dem Sirup dürfte somit auch die zweite theoretisch mögliche Form der racemischen 0xysäure vorhanden sein.

Hoppe-Seyler's Zeitschrift f. physiol. Chemie. IC. 
dem monoklinen System angehören. Die Säure löst sich leicht in Alkohol und Aceton, schwerer in Essigester, in Petroläther, Benzol und Chloroform ist sie fast unlöslich. $100 \mathrm{ccm}$ wasserhaltigen Äthers lösen $0,47 \mathrm{~g}$ der Lactonsäure. Eine 5\% ige wässerige Lösung derselben dreht das polarisierte Licht im $1 \mathrm{dm}-$ Rohr nicht. Gegenüber Kaliumpermanganat ist die sodaalkalische Lösung sehr beständig, während der Sirup, aus dem die Lactonsäure herauskrystallisierte, leichter angegriffen wird, woraus gefolgert werden kann, da $B$ derselbe neben der zweiten racemischen Form der $\gamma-0 x y-\beta-\gamma-\epsilon$-pentantricarbonsäure wahrscheinlich auch Itaconsäurederivate enthält, von denen theoretisch sechs verschiedene vorliegen könnten, neben drei Aticonsäurederivaten, von den möglichen cis- und trans-Formen abgesehen, welche Mannigfaltigkeit übrigens auch die Schwierigkeit erklärt, die krystallisierte Lactonsäure zu erhalten.

Verhalten der Lactonsäure beim Erhitzen. Wiedergewinnung von Hämatinsäure.

Beim Erhitzen der Lactonsäure im offenen Gefäß auf $138^{\circ}$ findet unter Braunfärbung ein Gewichtsverlust statt, der bei einem Versuch zu 5,21\% bestimmt wurde $(0,4072 \mathrm{~g}$ verloren in $2^{1 / 2}$ Stunden $0,0212 \mathrm{~g}$ ). Es dürfte sich um beginnende Anhydrisierung handeln (ber. für ein Molekül Wasser $8,91 \%$ ).

Steigert man das Erhitzen über den Schmelzpunkt, so beginnt bei $175^{\circ}$ Kohlendioxydentwicklung, die sich bei $195^{\circ}$ steigerte; bei $230-235^{\circ}$ destillierte dann ein Öl über, das sich als Methyläthylmaleinsäureanhydrid erwies. Bei der quantitativen Verfolgung dieser Reaktion wurde festgestellt, daß der Zerfall der Lactonsäure in Wasser und die angegebenen Zersetzungsprodukte sich nahezu vollständig vollzieht.

$0,332 \mathrm{~g}$ Lactonsäure gaben 0,06 Rückstand und $0,17 \mathrm{~g}$ des flüchtigen Öls, während sich die $\left.28,45 \mathrm{ccm}^{1 / 10-n-B a(O H}\right)_{2}$ entsprechende Menge Kohlendioxyd entwickelte.

Ber. für 0,272 $\mathrm{C}_{8} \mathrm{H}_{10} \mathrm{O}_{9}: 0,18 \mathrm{~g} \mathrm{C}_{7} \mathrm{H}_{8} \mathrm{O}_{3}$ und $0,06 \quad \mathrm{CO}_{2}$ Gef.: $\quad 0,17>\mathrm{C}_{7} \mathrm{H}_{8} \mathrm{O}_{3}>0,0626 \mathrm{CO}_{2}$. 
Über Synthese der Hämatinsäure und über 0xydation des Hämatins. 247

Das Öl wurde als Methyläthylmaleinsäureanhydrid durch den Siedepunkt nach Siwoloboffs Methode (gef. 227-228 unk.) und durch Titration charakterisiert: 0,0483 verbrauchten $\left.7,1 \mathrm{ccm}{ }^{1 / 10-n-B a(O H}\right)_{2}$ statt $6,9 \mathrm{ccm}$ (Phenolphthalein). Neben dem Anhydrid wurden im Destillat einige wenige Krystalle beobachtet, ein Befund, der in weiterer Verfolgung zur Auffindung eines Itaconsäurederivates führte.

$\mathrm{Da}$ aus dem geschilderten Versuch hervorging, daß die Zersetzung der Lactonsäure unter Kohlendioxydentwicklung erst bei $195^{\circ}$ stärker eintritt, war zu hoffen, daß ein Erhitzen nur bis zu dieser Temperatur zur Hämatinsäure zurückführen würde. In der Tat wurde ca. $1 \mathrm{~g}$ der Lactonsäure bei $11 / 2$ stündigem Erhitzen auf 180-195 ${ }^{\circ}$ bei gewöhnlichem Druck unter nur teilweiser Zersetzung in Hämatinsäure verwandelt. Man erhielt nach dem Erhitzen einen nach Methyläthylmaleinsäureanhydrid riechenden braunen Sirup, der im Lauf von 24 Stunden zu den für die Hämatinsäure charakteristischen Krystallformen erstarrte. Die Lösung desselben in $15 \mathrm{ccm}$ Wasser gab durch dreimaliges Ausschütteln an Chloroform $0,27 \mathrm{~g}$ Substanz ab, die sich als Hämatinsäure erwies, während $0,35 \mathrm{~g}$ im Wasser gelöst blieben.

Daß die Regenerierung der Hämatinsäure beim Erhitzen unter vermindertem Druck sehr glatt erfolgt, wurde bereits in den "Berichten» erwähnt.

Wiedergewinnung von Hämatinsäure durch Kochen der rohen Oxysäuren mit Laugen.

Die Lösungen der zu diesen Versuchen benutzten rohen Oxysäuren wurden mit Chloroform extrahiert, bis alle Hämatinsäure entfernt worden war, und mit Kalilauge gekocht, wonach die vorsichtig wieder angesäuerte Lösung erschöpfend mit Äther ausgezogen und der Rückstand der ätherischen Lösung, in Wasser gelöst, mit Chloroform extrahiert wurde. Hierbei wurden etwa $13 \%$ der verwendeten Rohsäure an Hämatinsäure wieder erhalten, deren Schmelzpunkt bei 97 bis $98^{\circ}$ lag. 


\begin{tabular}{c|c|c|c|c|c|c}
\hline Oxysäure & $\begin{array}{c}\text { Menge } \\
\text { der } \\
\text { Kalilauge } \\
\mathrm{g}\end{array}$ & $\begin{array}{c}\text { Prozent- } \\
\text { Gehalt } \\
\text { der Kali- } \\
\text { lauge } \\
\%\end{array}$ & $\begin{array}{c}\text { Dauer } \\
\text { des } \\
\text { Kochens } \\
\text { Std. }\end{array}$ & $\begin{array}{c}\text { Äther- } \\
\text { extrakt }\end{array}$ & $\begin{array}{c}\text { In Chloro- } \\
\text { form } \\
\text { löslich }\end{array}$ & $\begin{array}{c}\text { Prozent } \\
\text { an } \\
\text { Hämatin- } \\
\text { säure } \\
\%\end{array}$ \\
\hline 1,87 & 50 & 30 & 9 & 1,6 & 0,15 & 9 \\
1,45 & 50 & 40 & 9 & 1,33 & 0,18 & 13,4 \\
1,15 & 25 & 50 & 8 & 1,08 & 0,136 & 13,3
\end{tabular}

VI. Gewinnung des Itaconsäurederivats $\mathrm{C}_{7} \mathrm{H}_{10} \mathrm{O}_{4}$ aus der Hämatinsäure.

Wie bereits erwähnt, entsteht beim Erhitzen des Lactons der $\gamma$-Oxy- $\beta-\gamma-\epsilon-$ pentantricarbonsäure neben Methyläthylmaleinsäureanhydrid in Spuren eine flüchtige krystallisierende Substanz. In etwas besserer Ausbeute wurde sie bei der Destillation desselben $r$-Lactons bei $10 \mathrm{~mm}$ Druck und einer Badtemperatur von $230^{\circ}$ erhalten und von dem Anhydrid durch dessen Destillation mit Wasserdämpfen getrennt. Die Nichtflüchtigkeit der neuen Säure hierbei war jedenfalls darauf zurückzuführen, daß aus dem Anhydrid durch Wasseraufnahme die Säure entstanden war. Dieselbe hatte sich allem Anschein nach gebildet, als die $\delta-0 x y-\beta-\gamma-\epsilon-$ pentantricarbonsäure der Destillation unter vermindertem Druck unterworfen worden war. So stand zu erwarten, daß sie sich auch unter ähnlichen Bedingungen aus der Hämatinsäure selber bilden würde, denn die letztere entsteht ja aus den beiden Oxysäuren bei Temperaturen bis $190^{\circ}$, mußte also auch bei den Destillationen primär entstanden sein. Und in der Tat bildete sich die neue Säure aus Hämatinsäure. Die letztere destilliert bei einem Druck von $10 \mathrm{~mm}$ und einer Badtemperatur von 238-240 unter teilweiser Zersetzung.

Die Trennung der flüchtigen Produkte erfolgte durch Destillation mit Wasserdämpfen, wobei sich Methyläthymaleinsäureanhydrid verflüchtigt, Eindampfen der wässerigen Lösung auf ein geringes. Volumen, wobei sich manchmal die neue Säure bereits krystallisiert abscheidet, und Ausschütteln der Lösung mit Chloroform, welches Lösungsmittel die Hämatin- 
säure allein wegnimmt. Aus $1,5 \mathrm{~g}$ Hämatinsäure wurden im besten Fall bei solchen Versuchen gewonnen: 0,56 g destillierte Hämatinsäure, $0,23 \mathrm{~g}$ Methyäthylmaleinsäureanhydrid und $0,7 \mathrm{~g}$ der neuen Säure, die sich nach allen Eigenschaften als ein Itaconsäurederivat erwies. Zweimal aus heißem Wasser umkrystallisiert, zeigte sie gewöhnlich den Schmelz- und Zersetzungspunkt $179-180^{\circ}$, nachdem bei $170^{\circ}$ schwaches Sintern eingetreten war. Eine kleine Fraktion zeigte einmal rasch auf $175^{\circ}$ und dann in 3 Minuten auf $192^{\circ}$ erhitzt, erst bei dieser Temperatur die Zersetzung unter Schmelzen. ${ }^{1}$ )

Die Analyse wies auf die empirische Zusammensetzung $\mathrm{C}_{7} \mathrm{H}_{10} \mathrm{O}_{4}$.

0,1453 Substanz (i. V. obne Abnahme getrocknet): 0,2837 $\mathrm{CO}_{2}$ u. $0,0858 \mathrm{H}_{2} \mathrm{O}$.

$$
\begin{array}{ll}
\mathrm{C}_{7} \mathrm{H}_{10} \mathrm{O}_{4} \text {. } & \begin{array}{l}
\text { Ber.: } 53,16 \% \mathrm{C} \text { und } 6,33 . \% \mathrm{H} \\
\text { Gef.: } 53,25 \%
\end{array} \quad 6,56 \%
\end{array}
$$

Die Titration zeigte die Zweibasizität.

0,3300 Substanz erforderten 20,77 1/s-n- $\mathrm{Ba}(\mathrm{OH})_{2}$ (Phenolphthalein).

$\mathrm{C}_{7} \mathrm{H}_{10} \mathrm{O}_{4}$. Ber. 2 basisch : $20,88 \%$.

Die sodaalkalische Lösung der Säure wurde durch Kaliumpermanganat sofort entfärbt.

Krystallform. Aus der wässerigen Lösung scheiden sich beim Einengen körnige Krystalle ab, die, unter dem Mikroskop betrachtet, aus dicken, zu Drusen vereinigten Prismen bestehen. Aus der alkoholischen Lösung bilden sich lanzettförmige Krystalle, die zu sternförmigen Gebilden vereinigt sind.

Die Destillation unter $5 \mathrm{~mm}$ Druck ergab ein Destillat, aus dem etwa zu gleichen Teilen Methyläthylmaleinsäureanhydrid und uñveränderte Säure gewonnen wurde, bei gewöhnlichem Druck war nur wenig der letzteren vorhanden, vorwiegend das Anhydrid, geringe Kohlendioxyentwicklung trat auf. Dieses Verhalten stimmt mit den Angaben Fittigs bei Annahme der Itaconsäurestruktur für die neue Säure

1) Nach Fittig (Liebigs Ann. der Chem., Bd. 304, S. 122) fällt bei den Itaconsäuren Schmelz- und Zersetzungspunkt zusammen oder der letztere liegt tiefer, daher ist das Schmelzen nicht glatt, sondern findet je nach schnellerem oder langsamen Anheizen bei verschiedenen Temperaturen statt. 
überein, wonach die Destillation der Itaconsäurederivate unter gewöhnlichem Druck größtenteils Citraconsäure-Anhydride, im Vakuum fast nur Itaconsäureanhydride liefert.

VII. Oxydation des Hämatins, ${ }^{1}$ ) Gewinnung einer Säure, die beim Erhitzen Hämatinsäure liefert.

A. Oxydation von Hämin nach der von $R$. Willstätter angegebenen Methode, ${ }^{2}$ ) d. $h$. in schwefelsaurer Lösung mit Chromsäure, deren Menge etwa 29 Atomen Sauerstoff auf ein Molekül Hämin entspricht.

Die Ausbeute an Rohsäuren betrug: Hämins,

a) durch Ausschütteln mit Äther $37 \%$ des verwendeten

b) durch nachfolgende Apparat-Extraktion 9\% des verwendeten Hämins.

Die Verarbeitung der Rohsäuren geschah folgendermaßen: die wässerige Lösung wurde mit Soda alkalisch gemacht und ausgeäthert, nach dem Ansäuern folgte abermaliges Ausäthern, wodurch die gereinigte Rohsäure erhalten wurde. Diese wurde mit Barytwasser verseift und aus den gefällten Baryumsalzen nach Lösen in Salzsäure durch Chloroformextraktion die annähernd reine Hämatinsäure $\mathrm{C}_{8} \mathrm{H}_{8} \mathrm{O}_{5}$ erhalten. Hierbei wurden erhalten:

a) Ätherextrakt aus alkalischer Lösung $3 \%$ des verwendeten Hämins,

b) gereinigte Rohsäure $30 \%$ des verwendeten Hämins,

c) Hämatinsäure $\mathrm{C}_{8} \mathrm{H}_{8} \mathrm{O}_{5}$ ca. $18 \%$ des verwendeten Hämins. Aus der mittels Apparatauszuges gewonnenen Rohsäure wurden nur ganz geringe Mengen $\mathrm{C}_{8} \mathrm{H}_{8} \mathrm{O}_{5}$ erhalten.

1) Die Oxydation des Hämatins ist des öfteren von mir beschrieben worden (Biese Zeitschrift, Bd. 44, S. 391 [1905]); da die von Ha as und mir erübrigten guten Ausbeuten an Hämatinsäure nicht immer bestätigt werden konnten, veranlaßte ich Herrn Weller, bei seinen Versuchen mit größter Sorgfalt die Ergebnisse zu verfolgen. 'In der Tat hat er nun geringere Ausbeuten zu verzeichnen, was zum Teil auf die Art der Oxydation zurückzuführen ist, z. T. aber sicher darauf, daß Herr Weller meist alte Hämin- oder Hämatinpräparate verarbeitet hat. W. Küster.

2) Liebigs Ann. d. Chemie, Bd. 373, S. 227 (1910). 
Die nach Verseifung und Entfernung des überschüssigen Baryts durch Einleiten von Kohlendioxyd in die heiße Lösung gelöst gebliebenen Baryumsalze wurden durch Alkohol gefällt. Ihre Menge betrug $16 \%$ des verwendeten Hämins. Die Baryumsalze des Schüttelextraktes zeigten nach dem Trocknen bei $100^{\circ}$, wobei ein Gewichtsverlust von 6-8\% eintrat, einen Gehalt von $48,7 \% \mathrm{Ba}$, während das lösliche Baryumsalz des Apparatextraktes beim Trocknen 10\% verlor und nur $45,5 \%$ enthielt.

Aus den löslichen Baryumsalzen des Schüttelextraktes wurde nach Zerlegung mittels Schwefelsäure durch Chloroformextraktion Hämatinsäure, durch nachfolgende Ausätherung Bernsteinsäure erhalten, d. h. die Extrakte bestanden nicht etwa aus den reinen Säuren, sondern die im sirupösen Extrakt eingebetteten Krystalle zeigten nach der Reinigung durch Abpressen und Befeuchten mit Äther auf Ton bezw. durch Umkrystallisieren aus heißem Wasser die Schmelzpunkte der genannten Säuren. Sowohl aus dem Chloroformextrakt, als auch aus dem Ätherextrakt wurden nach Entfernung der Krystalle wieder Baryumsalze hergestellt (durch Kochen mit Barytwasser, Einleịten von Kohlendioxyd, Fällung des Filtrates mit Alkohol). Nun erhielt das Baryumsalz des ersteren 47,7\% Ba.; das des Ätherextrakts 49,7. Aus jenem wurde Hämatinsäure, aus letzterem dann wieder Bernsteinsäure erhalten. Es hatte sonach den Anschein, als ob die im löslichen Baryumsalz enthaltene Säure bei der Einwirkung von Säuren immer teilweise in Hämatin- und in Bernsteinsäure zerfalle in Übereinstimmung mit früheren Beobachtungen.1)

1) Vgl. diese Zeitschr., Bd. 44, S. 420 (1905); Bd. 59, S. 94 (1909).

Um ein Gemisch der beiden Säuren konnte es sich nicht handeln. Das Baryumsalz der Hämatinsäure löst sich in heißem Wasser nur zu etwa $0,25 \%$, das der Bernsteinsäure zu etwa 0,35\%. Wird eine bei Zimmertemperatur bereitete Lösung, welche beide Baryumsalze enthält, gekocht, so blieb in $150 \mathrm{~g}$ des Filtrates vom ausgeschiedenen Baryumsalz $1 \mathrm{~g} \mathrm{Salz} \mathrm{gelöst,} \mathrm{das} \mathrm{höchstens} \mathrm{zu} \mathrm{einem} \mathrm{Drittel} \mathrm{aus} \mathrm{Baryumhämatinat}$ bestand. Die Löslichkeit desselben war also durch das Succinat nicht erhöht, sondern eher vermindert worden. Ein gleiches Verhalten wurde bei den Kupfersalzen festgestellt.

Auch ist die Trennung von Hämatinsäure und Bernsteinsäure über 
B. Oxydation des Hämatins in essigsaurer Lösung mit Chromsäure in einer 21 Atomen Sauerstoff auf das Molekül Hämatin entsprechenden Menge. Die Rohsäure wurde zur Entfernung flüchtiger Stoffe mit Wasserdämpfen behandelt, wobei auch Abscheidung harziger Stoffe eintrat. Alsdann wurde direkt verseift, da bei dieser Methode nur äußerst geringe Mengen aus alkalischer Lösung in Äther überzugehen pflegen. Die Ausbeuten betrugen:

A. an gereinigter, d. h. von Harz und flüchtigen Stoffen befreiter Rohsäure $51-53 \%$ des verwendeten Hämins.

B. a) aus den unlöslich gewordenen Baryumsalzen

a) durch Chloroformextraktion: 24\% vom verwendeten Hämin;

B) durch Ätherextraktion: 3\%;

b) lösliche Baryumsalze: 32\% vom verwendeten Hämin.

Eine dritte Reihe von Oxydationsversuchen wurde in der Weise vorgenommen, daß das Hämatin zunächst in essigsaurer Lösung mit einer nur zwölf Atomen Sauerstoff entsprechenden Menge Chromtrioxyd versetzt wurde und das bei der Aufarbeitung zur Gewinnung der ätherlöslichen Säuren abfallende in Äther unlösliche Oxydationsprodukt des Hämatins von neuem und zwar mit $3 / 4$ der zur ersten Oxydation verwendeten Menge von Chromsäure oxydiert wurde, nachdem es in 50 gewichts$\%$ iger Schwefelsäure gelöst worden war. Hierbei wurde eine Temperatur von $5-10^{\circ}$ eingehalten.

Die Ausbeuten betrugen hierbei:

A. fast $40 \%$ gereinigte Rohsäure.

B. a) a) $15,3 \%$ Chloroform-, $\beta$ ) ca. $1 \%$ Ätherextrakt.

B. b) fast $33 \%$ lösliche Baryumsalze. ständen.

C. $60 \%$ des verwendeten Hämins an Oxydationsrück-

Die schwefelsaure Lösung der letzteren wurde, bevor

die Baryum- oder Kupfersalze der Trennung durch Chloroform keineswegs vorzuziehen. Das Kupferhämatinat ist zwar bei Zimmertemperatur bedeutend leichter löslich als das Succinat, das sich nur zu 0,03\% löst, durch Erhitzen der Lösungen oder Zugabe von Kupferhydroxyd bilden sich aber bei beiden Säuren schwerer lösliche basische Salze. 
Uber Synthese der Hämatinsäure und über Oxydation des Hämatins. 253

sie weiter oxydiert wurde, mit Äther extrahiert, wobei noch ungefähr 5\% gereinigte Rohsäure gewonnen wurde. Nach vollzogener Oxydation wurden erhalten:

A. ca. $27 \%$ gereinigte Rohsäure.

B. a) a) ca. $2 \%$ Chloroform-, $\beta$ ) ca. 2,4\% Ätherextrakt;

b) $31,6 \%$ lösliche Baryumsalze,

wozu noch bemerkt sei, daß viel flüchtige Stoffe entstanden waren, unter anderen Ameisensäure und ein neutraler reduzierend wirkender Stoff, und ferner Oxalsäure. Die Ausbeuten an Hämatinsäure (Chloroformextrakt) waren also bei diesem Verfahren am geringsten, und es ist also weit zweckmäßiger, die ganze zur Oxydation des Hämatinmoleküls benötigte Menge von Chromtrioxyd, die 21 Atomen Sauerstoff zu entsprechen hat, auf einmal zuzugeben, was mit früher gemachten Erfahrungen übereinstimmt.

Untersuchung der leicht in Wasser löslichen Baryumsalze.

Sie erwiesen sich nach dem Verdampfen ihrer Lọsung zur Trockne nur noch zum Teil als in heißem Wasser löslich; von $17 \mathrm{~g}$, welche von der 0xydation des Hämatins herrührten, gingen z. B. nur noch $13,5 \mathrm{~g}$ leicht in Lösung, von $8,4 \mathrm{~g}$, die bei der Oxydation der Rückstände gewonnen waren, 5 g. Die unlöslichen Teile gaben nach der Zerlegung mit Salzsäure an Chloroform erhebliche Mengen von Hämatinsäure, an Äther wenig Bernsteinsäure ab.

Die löslich gebliebenen Teile wurden ebenfalls mit Salzsäure zerlegt und die Lösungen im Extraktionsapparat zunächst mit Chloroform, dann mit Äther extrahiert, wobei die Ausbeuten an Säuren weit hinter den berechneten zurückstanden, wahrscheinlich war viel zu wenig Salzsäure verwendet worden, da allem Anschein nach Oxysäuren vorliegen, die erst aus stark salzsäurehaltiger Lösung in Äther übergehen. ${ }^{1}$ )

Erhalten wurden aus

13,5 g lösl. Baryumsalz 0,78 Chloroform- und 3,5 g Ätherextrakt

5 , , , $0,45,1,57$, ,

i) Vgl. S. 239. 
Von diesen enthielten schon die ersteren nur sehr wenig Hämatinsäure, die Ätherextrakte waren frei von dieser Säure. Nach monatelangem Lagern zeigten sich Krystalle, die als Bernsteinsäure identifiziert wurden; es lag daher nahe, auch auf Hämatinsäure zu prüfen, da ein abermaliger Zerfall einer kompliziert gebauten Säure in diese beiden Bestandteile nicht unwahrscheinlich war. Doch lieferte eine erneute Extraktion der wässerigen Lösung des von der Bernsteinsäure befreiten Sirups durch Chloroform keine Hämatinsäure. So wurde die Lösung wieder mit Äther ausgezogen, was einigermaßen vollständig erst gelang, als Salzsäure bis zu einer Konzentration von 20\% hinzugegeben worden war. Der Rückstand der ätherischen Lösung im Betrage von 1,5 $\mathrm{g}$ wurde nunmehr eine Stunde lang auf 172 bis $181^{\circ}$ erhitzt, nach dem Erkalten in Wasser gelöst und diese Lösung mit Chloroform extrahiert, wobei $0,36 \mathrm{~g}$ herausgelöst wurden. Der Rückstand der wässerigen Lösung (0,8 g), zum zweiten Male erhitzt, ergab dann noch $0,13 \mathrm{~g}$ Chloroformextrakt. Die vereinigten Chloroformextrakte wurden durch Aufnehmen in Äther gereinigt, wobei ein harziger Körper zurückblieb, der Rückstand der ätherischen Lösung in Wasser aufgenommen, mit Baryt neutralisiert und die Lösung gekocht, wobei eine Fällung auftrat. Nach dem Zerlegen derselben durch Salzsäure wurde wieder mit Äther ausgeschüttelt und jetzt $0,174 \mathrm{~g}$ Säure gewonnen, die sehr bald in den Formen der Hämatinsäure zu krystallisieren begann. Ein reines Präparat lag aber jetzt noch nicht vor, da statt der berechneten $14,17 \mathrm{ccm} \quad 1 / 5-n$-Barytwasser nur $13,43 \mathrm{ccm}$ verbraucht wurden. Aus dem beim Kochen dieser Baryumsalzlösung gefällten Baryumsalz wurde dann aber Hämatinsäure erhalten, die nach dem Umkrystallisieren bei $96^{\circ}$ schmolz. Damit ist nun äußerst wahrscheinlich geworden, daß sich bei der Oxydation des Hämatins Oxysäuren bilden, die beim Erhitzen unter Wasserabspaltung Hämatinsäure liefern. Sie dürften aus prinär gebildeter Hämatinsäure durch Anlagerung von Wasser unter dem Einfluß der Schwefelsäure entstanden sein, die bei der Aufarbeitung der die Oxydationsprodukte des Hämatins enthaltenden Flüssigkeiten benötigt wurde. 